\title{
Will Enhanced Depth-of-focus Intraocular Lenses \\ Lead the Future in Premium Lenses?
}

\author{
Aylin Kılıç \\ Cataract and Refractive Surgeon, Dunya Eye Hospital, Istanbul, Turkey
}

DOI: http://doi.org/10.17925/EOR.2015.09.02.100

\begin{abstract}
Multifocal intraocular lenses (MF IOLS) continue to undergo modifications to enhance distance and near vision. Extended range of vision IOLS do not have multiple focal points as other MF IOLs do, so they do not cause any disturbance in distance vision and can compensate for chromatic aberrations.

\section{Keywords}

Chromatic aberration, distance vision, near vision, pupil size

Disclosure: Aylin Kiliç has nothing to disclose in relation to this article. No funding was received in the publication of this article.

Open Access: This article is published under the Creative Commons Attribution Noncommercial License, which permits any non-commercial use, distribution, adaptation and reproduction provided the original author(s) and source are given appropriate credit.

Received: 23 October 2015 Published Online: 21 December 2015 Citation: European Ophthalmic Review, 2015;9(2):100-1

Correspondence: Aylin Kilıç, Dunya Eye Hospital, Istanbul, Turkey. E: aylinkilicdr@gmail.com
\end{abstract}

Presbyopia normally develops as a person ages and is associated with a natural progressive loss of accommodation ability to rapidly and easily refocus on objects at varying distances. A variety of technologies have been developed to enhance the ability of the intraocular lens (IOL) to facilitate viewing. Multifocal (MF) IOLs were designed to eliminate the need for spectacles after cataract surgery. Traditional MF IOLS use the principles of refraction, diffraction or a combination of both to divide the incoming light into two or three focal points: near, intermediate and distance. ${ }^{1}$ MF diffractive optical surface directs portions of the light energy towards differing focal distances. Diffractive optical surfaces, either monofocal or MF, may also have aberrations. In contrast to refractive IOLS, diffractive MF IOLs use a system of concentric rings to bend lights into focal points simultaneously: one for distant vision; the other for near vision. Diffractive IOLs are largely pupil independent, meaning that the MF abilities of IOLs are less independent upon pupil size., ${ }^{2,3}$

A new segment of IOLS, known as the extended range of vision IOLS, were designed to provide good vision at a range of distances. The TECNIS ${ }^{\circledR}$ Symfony (Abbott Laboratories Inc., Illinois, US) IOLS include an echelette design feature. Echelettes are annular diffractive lens zones spaced about the optical axis of the lens. The surface area or diameter of the echelettes determines the diffractive powers of the $\mathrm{IOL}$ and the step height of the transition between echelettes determines the light distribution between the different add powers. This echelette profile is designed such that it shifts the wavelength of light and causes increased depth of focus. This technology combines two enabling proprietary technologies to simultaneously extend the range of vision and improve the contrast. Proprietary echelette design elongates the focus of the eye, resulting in an extended range of vision, and proprietary achromatic technology has been designed to reduce chromatic aberration, resulting in enhanced contrast sensitivity. ${ }^{4}$

\section{Chromatic Aberration}

The idea behind chromatic aberration correction is simply to get the colours of the spectrum focused at the same point. Chromatic aberration is mainly induced by the IOL optic material and this term is used to describe an alteration of colour. ${ }^{5}$ The Abbe number of a material indicates the amount of chromatic aberration caused by a lens material. It is important to use an IOL material with a high Abbe number since studies have shown that chromatic aberration from IOLs may negatively impact visual acuity, contrast sensitivity and functional vision. ${ }^{6}$

Chromatic aberration can be compensated by an achromatism lens in front of the eye with an IOL. The highest improvement in visual performance is obtained when both chromatic aberration and spherical aberration are corrected to cause enhancement of contrast. ${ }^{7.8}$

\section{Contrast Sensitivity}

Modular transfer function (MTF) is a measure of the amount of contrast transferred by the optics in a visual system. The higher the MTF, the more contrast is transferred from the original object to the image formed on the retina, and consequently the perceived image. ${ }^{9}$ The TECNIS Symfony IOL has high MTF50 for $5 \mathrm{~mm}$ in the ACE model, so this causes enhanced contrast sensitivity.10

Standard MF IOLS can reduce contrast sensitivity when compared with monofocal IOLs under low-contrast conditions, such as driving at night or in poor visibility conditions. This is due to the fact that they divide the incoming light into two focal points, often reducing the light used to form an image to less than $50 \%$. Ultimately, this reduces MTF values. ${ }^{11}$ The optic material of this lens corrects both spherical and chromatic aberration, providing a high-quality retinal image. Pupil independence is also a function of optics and lens material, not necessarily lens design. 
Glistenings have also been reported to cause a loss in contrast sensitivity. IOLs with no glistenings were related to a $40 \%$ increase in contrast sensitivity at high spatial frequencies, which was not reported with the other IOLS on the TECNIS platform. ${ }^{12}$

Multiple clinical studies show that TECNIS Symfony IOL patients experienced high spectacle independence for distance, intermediate and near vision. The TECNIS Symfony IOL provides 20/20 or better mean uncorrected and best corrected distance, distance-corrected intermediate visual acuities and 20/30 mean distance corrected near visual acuity. Extended range of vision IOLs also shows a clinically significant increase in depth of vision by providing $20 / 20$ or better vision up to -1.5 D of defocus and $20 / 40$ or better vision up to -2.5 $\mathrm{D}$ of defocus. Defocus curves demonstrate the tolerance to residual refractive errors, meaning that the patient can have up to $1.5 \mathrm{D}$ of hyperopia and still maintain the same distance vision; however, this would compromise an improvement for intermediate and near vision. ${ }^{33}$

The extended range of vision IOL is a new segment in presbyopia-correcting IOLS with a significant advancement over traditional technologies: full range of high-quality continuous vision, no incidence of halos comparable with a monofocal IOL and enhanced contrast sensitivity.
1. Cochner B, Lafuma A, Khosnood B, et al., Comparison of outcomes with multifocal intraocular lenses: a meta-analysis, Clin Ophthalmol, 2011:5:45-56.

2. Walkow T, Liekfeld A, Anders N, et al., A prospective evaluation of a diffractive versus a refractive designed multifocal intraocular lens, Ophthalmology, 1997;104:1380-6.

3. Montés-Micó R, España E, Bueno I, et al., Visual performance with multifocal intraocular lenses: mesopic contrast sensitivity under distance and near conditions, Ophthalmology, 2004;111:85-96.

4. TECNIS Symfony DFU, Tecnis Symfony Extended Range of Vision IOL DFU Z310939 Rev. 03, Santa Ana, CA: Abbott Medical Optics, Inc., 2014.
5. Friedman N, Kaiser P, Basic optics, In: Essentials of Ophthalmology, 1st ed., Friedman N, Kaiser P, Philadelphia, PA: Saunders Elsevier, 2007;19-25.

6. Zhao H, Mainster MA, The effect of chromatic dispersion on pseudophakic optical performance, Br J Ophthalmol, 2007:91:1225-9.

7. Piers PA, Fernandez EJ, Manzanera S, et al., Adaptive optics simulation of intraocular lenses with modified spherica aberration, Invest Ophthalmol Vis Sci, 2004;45:4601-10.

8. Weeber HA, Piers PA, Theorical performance of intraocular enses correcting both spherical and chromatic aberration, $J$ Refract Surg, 2012;28:48-52.

9. Root T, Optics, In: Opthobook, Root T, Seattle, WA: Create
Space, 2009:117-26.

10. DOF2015CTO020 MTF of TECNIS Symfony IOL, and the other lens models, 29 June 2015, Tecnis Symfony IOL Dfu-ZXR-ZXTZ310939-Rev 05.REF2015CT0326.

11. Santhiago MR, Wilson SE, Netto MV, et al., Modulation transfer function and optical quality after bilateral implantation of $\mathrm{a}+3.00 \mathrm{D}$ versus $\mathrm{a}+4.00 \mathrm{D}$ multifocal intraocular lens, $J$ Cataract Refract Surg, 2012;38:215-20.

12. Gunenc $\mathrm{U}$, Oner $\mathrm{FH}$, Tongal $\mathrm{S}$, et al., Effects on visual function of glistenings and folding marks in Acrysof intraocular lenses, J Cataract Refract Surg, 2001;27:1611-4.

13. Clinical Investigation of a Design Extension of The Tecnis 1-Piece Intraocular Lens, Santa Ana, CA: AMO, 2014. 